\title{
No hay diferencias a largo plazo entre mastectomía radical versus mastectomía total con y sin radioterapia
}

Twenty five year follow-up of a randomized trial comparing radical mastectomy, total mastectomy, and total mastectomy followed by irradiation. Bernard Fisher, M.D, Jong-Hyeon Jeong, Ph.D., Stewart Anderson, Ph.D.N England J Med. 2002,August 22;347:567-575.

\section{Objetivo}

Determinar la evolución a largo plazo de distintas estrategias terapéuticas en pacientes con cáncer de mama.

Diseño

Ensayo aleatorizado con seguimiento promedio de 25 años.

\section{Lugar}

Estudio multicéntrico, 33 centros en Estados Unidos y uno en Canadá.

\section{Pacientes}

Fueron incluidos en el estudio 1765 pacientes con cáncer de mama primario operable. Más del $70 \%$ tenía 50 o más años al inicio del estudio (1971 a 1974) y un tamaño promedio del tumor de $3.3 \mathrm{~cm}(+/-2 \mathrm{~cm})$ en el subgrupo sin compromiso de los ganglios linfáticos axilares y $3.7 \mathrm{~cm}(+/-2 \mathrm{~cm})$ en quienes sí lo tenían.

\section{Intervención}

Se aleatorizaron 1665 pacientes según el compromiso linfático (clínicamente positivo o negativo).

Axila positiva: dos grupos.

1. Mastectomía radical (292).

2. Mastectomía total + terapia radiante (294).

Axila negativa: tres grupos.

1. Mastectomía radical (362).

2. Mastectomía total (365)

3. Mastectomía total + terapia radiante (352).

Fue empleada la radioterapia por supervoltaje con una dosis de 5000 rads (50Gy actuales) a través de 25 fracciones en las axilas negativas y se realizó un refuerzo adicional de 10 a 20Gy en las positivas. Se utilizó además una dosis de 45Gy en la cadena mamaria interna y supraclavicular y, además, campos tangenciales de 50Gy en la pared torácica.

\section{Medición de resultados principales}

Los puntos finales comparativos entre grupos fueron: 1) sobrevida libre de enfermedad, 2) intervalo libre de recidiva, 3) sobrevida libre de enfermedad a distancia, 4) sobrevida global.

\section{Resultados principales}

No se observaron diferencias entre los tres grupos de mujeres sin compromiso axilar, ni tampoco entre los dos grupos de mujeres con ganglios axilares positivos con respecto a la sobrevida libre de enfermedad, el intervalo libre de recidiva, la sobrevida libre de metástasis y la sobrevida global,

En las mujeres sin compromiso axilar, el riesgo relativo de muerte entre aquellas tratadas con mastectomía total más radioterapia y las que recibieron mastectomía radical fue de 1.08 (IC95\%: 0.91 a 1.28; $p=0.38$ ) y entre las que efectuaron mastectomía total sin radioterapia y las sometidas a mastectomía radical, de 1.03 (IC95\%: 0.87 a $1.23 ; p=0.72$ ).

El riesgo relativo de muerte entre las mujeres con compromiso axilar que efectuaron mastectomía total más radioterapia y aqueIlas que fueron mastectomizadas en forma radical fue de 1.06 (IC95\%: 0.89 a $1.27 ; p=0.49$ ).

\section{Conclusiones}

Este estudio sugiere, al iqual que informes preliminares, que no hay ventajas estadísticamente significativas en el uso de la mastectomía radical versus la mastectomía total.

Los hallazgos no demuestran una mejoría de la sobrevida a consecutiva a la extirpación de ganglios axilares positivos ocultos o por el uso de la radioterapia en el momento de la cirugía inicial.

Fuente de financiamiento: National Cancer Institute EUA.

\section{Comentario}

Los resultados de este estudio no pueden ser extrapolados de manera general debido, entre otras causas, al pequeño número de participantes enrolados. Sin embargo, en un metaanálisis ${ }^{1}$ otros grupos encontraron similares resultados respecto de la escasa mejoría en la sobrevida a los 20 años de tratamiento con el uso de la radioterapia.

Si bien hay una disminución de dos tercios en la probabilidad de recurrencia local, si son corregidas las cifras por el daño causado con la radioterapia, se ve una mejoría del 2 al $4 \%$ en la sobrevida global, lo que ajustado según la edad del tratamiento reduce este beneficio en pacientes jóvenes y lo aumenta en las de mayor edad.

Con los resultados de este estudio se pretendía sustituir a la mastectomía de Halstead como tratamiento estándar en el tratamiento del cáncer de mama. Esto dió paso en los años se-

tenta a posteriores trabajos ${ }^{2,3}$ en los que se comparó a la misma con la cuadrantectomía sola o con el tratamiento con radioterapia combinada, logrando cambios fundamentales en el planteo quirúrgico. Esto se basa en el hecho demostrado, en este estudio y en otros, de que el control local o de la recidiva local no influyen en el pronóstico global del cáncer de mama, que sí puede verse afectado por la existencia de focos ocultos a distancia o metástasis. Algunos estudios más recientes ${ }^{4,5,6}$ difieren con los resultados obtenidos en este trabajo, probablemente debido al uso de tratamientos adyuvantes como la quimioterapia, ausentes en el mismo por el enfoque principalmente quirúrgico de este planteo inicial.

Conclusión del comentario: A pesar del escaso o nulo impacto en la sobrevida global que han mostrado los resultados de este trabajo, no se debe subestimar el control local del cáncer de mama, siempre respetando el enfoque sistémico actual.

\section{Dr. Daniel Wajsfeld [ Servicio de Ginecologia del Hospital Italiano de Buenos Aires ]}

\footnotetext{
Referencias

1. Early Breast Cancer Trialist's Colaborative Group. Favourable and unfavourable effects on long-term survival of radiotherapy for early breast cancer: an overview of the randomized trials. Lancet 2000;355:1757-1770

2. Umberto Veronesi,M.D., Natale Cascinelli,M.D., Luigi Mariani,M.D, et al. Twenty -year follow-up of a randomized study comparing breast-conserving surgery with radical mastectomy for early breast cancer. N. Engl. J. Med 2002;347:1227-1232.

3. Bernard Fisher, M.D., Stewart Anderson, M.D., Ph.D., John Bryant, et al. Twenty-year follow-up of a randomized trial comparing total mastectomy, lumpectomy, and lumpectomy plus irradiation for the treatment of invasive breast cancer.

4. Overgaard M, Hansen PS, Overgaard J, et al. Postoperative radiotherapy in high-risk premenopausal woman with breast cancer who receive adjuvant chemoteraphy. N Engl. J. Med 1997;337:949955.

5. Overgaard M, Jensen M-B, Overgaard J, et al. Postoperative radiotherapy in high-risk postmenopausal breast-cancer patients given adjuvant tamoxifen: Danish Breast Cancer Cooperative Group DBCG 82C randomized trial. Lancet 1999;353:1641-1648:

6. Ragaz J, Jackson SM, L N, et al. Adjuvant Radiotherapy and chemotherapy in node-positive premenopausal women with breast cancer. N Engl. J. Med 1997;337:956-962.
} 